\title{
Player Experience Evaluation: An Approach Based on the Personal Construct Theory
}

\author{
Francesco Bellotti, Riccardo Berta, Alessandro De Gloria, and Ludovica Primavera \\ DIBE - Department of Electronics and Biophysical Engineering \\ University of Genoa, via Opera Pia 11 a, \\ 16145 Genova, Italy \\ \{franz, berta, adg, primavera\} @elios.unige.it
}

\begin{abstract}
The scientific and industrial community related to the videogame (VG) research and business is ever more concerned about the need for proper evaluation and assessment of games. This paper proposes an assessment methodology based on the Personal Construct Theory (PCT). The PCT allows identifying constructs can be processed to define a space where domain-relevant items - VGs, in our case - can be positioned. The main praise of PCT is that the test-leading researcher does not supply users with a predefined set of constructs, which may bias the evaluation process. Moreover, PCT joins qualitative aspects with a quantitative evaluation of their relevance, which is particularly useful for an operational approach also to game design. In this paper, we study the application of the PCT to the particular case of the evaluation of whole typologies of VGs. Discussing the results, we draw and highlight that VGs are perceived as engaging challenges where personal abilities are continuously put to the test. This stresses the reactive nature of VGs and the fact that players like being stimulated and developing and testing their reaction capabilities.
\end{abstract}

Keywords: Videogames, User Experience, videogame testing and evaluation, Repertory Grid Technique, Personal Construct Theory.

\section{Introduction}

Videogaming has acquired a great relevance since years, not only for the youngsters. Serious games applications expand the horizons of games to include scientific simulation and visualization, industrial and military training, medical and health training and education, as well as public awareness and policy change [1].

In the Transformation Economy people is expected to pay to have experiences that are transformational [2], and games and simulations allow significantly extending the range of experiences a person can live, with an ever growing level of realism. Producing a videogame (VG) has become a huge and expensive project, since the market is characterized by trends toward hit products and fewer Stock-Keeping Units [3]. In this scenario, awareness has risen of the importance of evaluation methodologies able to support producers and evaluators in assessing products since early phases of a project. However, there are not commonly shared and accepted techniques, yet [4]. 
In this paper we propose and discuss a VG evaluation methodology based on the Personal Construct Theory (PCT) of personality [5], which aims at determining an idiosyncratic measure of personality. The theory has been initially applied in psychological clinic interviews [6] and then in a number of other fields, also including website evaluation [7]. The PCT assumes that individuals perceive and evaluate the world through similarity-dissimilarity poles, called "personal constructs". Such poles can be used to define an individual's personal construct system relevant to the analyzed domain - in our case the evaluation of VGs. The main praise of PCT is that the testleading researcher does not supply users with a predefined set of constructs, which may bias the evaluation process [8]. Rather, constructs are defined a posteriori, based on an analysis of the free user comments. This is particularly promising for $\mathrm{VG}$ evaluation, because it stresses the centrality and relevance of the player experience, which is a fundamental value for a game success. Moreover, the theory gives indications based on the subjects' free evaluation of their domain (i.e. VG) experiences, which may give also ideas for new products, not only the evaluation of existing ones. PCT joins qualitative aspects - the elicited personal constructs - with a quantitative evaluation of their relevance, which is particularly useful for an operational approach also to game design.

We study the application of the PCT to the particular case of the evaluation of whole typologies of VGs (e.g. Sports, Racers, First Person Shooters). In particular, we intend to explore what the personal constructs are that define the space of the games according to test player experiences - and observe how current game typologies are placed with respect to such dimensions (i.e. the constructs). Finally, we also try to see if new, "hybrid" game typologies could be devised based on the combination of important constructs.

The paper is organized as it follows. Section 2 presents the main general aspects of PCT, section 3 provides a literature review of VG evaluation methodologies. Section 4 describes the procedure we have defined for the evaluation of player experience. The section 5 discusses the results, proposing observations and findings.

\section{The Personal Construct Theory (PCT)}

Personal Construct Psychology (PCP), also referred as Personal Construct Theory (PCT), originated with the pioneering work of George Kelly [9]. Kelly's theory of personality was based on the metaphor of the Inquiring Man [5]. A person is seen as a scientist who tries to make sense of the world by continually forming hypotheses about the world and experimenting and observing. The PCT assumes that the individual perceives and evaluates the world (e.g. other individuals, situations, etc.) through similarity-dissimilarity poles, called Personal Constructs. The meaning of any construct is defined by the complementarities of opposite poles, in Kelly words: "the differences expressed by a construct are just as relevant as the likenesses." [9]. The personal construct is the elementary unit that a person uses during her process of construction of meaning about the world. Thus, constructs represent the personal view of the world the subject has constructed based on her own experience. 


\subsection{The Repertory Grid Technique}

The Repertory Grid Technique (RGT) [10] is a methodological extension of PCT to empirically eliciting and evaluating individual's subjective personal construct system relevant to a topic. An RGT is a table in which rows contain constructs and columns contain elements (instances of the topic under investigation). To fill the table, the person refers to a rating system to quantitatively assign each element to one of the poles of each qualitative constructs. A grid is constructed for each subject participating in a test session. A subject produces his constructs list composed of bipolar dimensions that the person sees as important for talking about the elements. The elicitation process is based on the use of triads of elements. Each subject is presented with triads of such elements and asked to indicate in what respect two of the three are similar to each other and differ from the third one. These similarity/differentiation factors are recorded as bipolar constructs. Different triads may give rise to the similar (or even same) bipolar properties. Then, after having identified his individual constructs, the subject is asked to rate the degree to which each element is in relation to each bipolar construct, according to a Likert-type scale. The personal constructs can be then analyses and summed together, and the most frequent ones are assigned a higher weight as they represent the most appropriate properties able to characterize the domain. All such bipolar constructs (each one with a different weight) are thus elicited as the proper dimensions (or classification modes) along which the domain elements are evaluated by the user.

The main feature of RGT is that the researcher does not supply users with a predefined set of constructs, which may bias the evaluation process [10]. Rather, constructs are defined by the participants, based on the analysis (triad methods) of his comments.

\section{Literature Review}

The main feature of a successful VG is the enjoinment of the player, but there is no commonly accepted model for player enjoyment [4]. In literature there are several heuristics for designing and evaluating games, and all of them are focused on three main aspects: gameplay, mechanics and interface [11]. These heuristics are often fragmented and contradictory, because doesn't exist evidence about the relevance of used criteria versus VGs enjoinment [12]. From another point of view, many researchers have proposed models based on psychological theories, including disposition theory [13], attitude [14], transportation theory [15], para-social interaction [14] and flow [16]. The main drawback of these attempts is their predetermined structure [17]. Examples include methodology based on exploratory interview, which has the problem of imposition of terminology that prevents the participant to make use of their proper terminology in the discussions [18]. It is needed to combine these elements into a set of dimensions not predetermined a priori, but extracted from real users. Our approach, based on the RGT is focused on eliciting dimensions that are meaningful to the participant and not only to the experimenter. The data that we capture from end users has not been influenced by the researcher's theory. Moreover, it takes also into account users' idiosyncratic views in contrast to the other approaches, which impose a priori defined dimensions. 
Although the RGT technique was initially developed in psychological clinical settings in order to determine an idiosyncratic measure of personality, it has been applied to a wide variety of cases, including evaluation of user experience [8], also in the user experience evaluation research [19] [20]. Based on this analysis and our experience, we believe that RGT facilitates an objective approach to capturing subjective aspects of VG evaluation by allowing an open, broad and sensitive analysis of the several aspects that might have an impact on the user's experience of a VG.

Others techniques attempt to model user enjoinment based on intrusive measurements of user's physiology [8]. Example of these are based on facial electromyography as a measure of positive and negative emotional states [21], or on the children's heart rate signals for capturing and modeling individual entertainment preferences [22].

\section{Applying the Method to the Player Experience Evaluation}

This section describes the methodology, based on RGT, which we have defined and followed for VG evaluation, while the next session presents the results. A test session consists of three major steps: initial focus (users are asked to think about their concrete experience with items in the investigated domain), construct elicitation (users have to identify similarities and differences among elements/items in order for researchers to identify bipolar constructs), rating (users have to rate each element along each elicited construct). We describe in detail the following steps, but first we describe the test user selection procedure.

\subsection{Subject Selection}

In order to test our RGT to VGs, we have recruited test subjects by contacting teachers of three different high-schools with scientific and technical background. To each school we proposed a one-day workshop about VGs. We tested a total of 64 students in an age range from 16 to 19 (mean 17,4; stddev 0,64). There were 13 females and 51 males. We asked for students with a strong interest for VGs, as we wanted to have opinions from experienced users, who have a good familiarity with state of the art VGs. This subject selection criterion should allow us to have a realistic analysis of the current VG panorama. Of course, a similar analysis could be conducted on a more generalist population sample, for example if we were interested in understanding on how to attract new typologies of users (e.g. women). But this is not the specific purpose of this work. In a pre-test questionnaire phase we had tested the users' level of familiarity with VGs, in order to check the appropriateness of our target group. As expected, participants were quite familiar with VGs (mean 2,56; stddev $1,15)$ and would usually spend a huge amount of time playing with consoles and PCs (1-3 hours per day).

\subsection{Initial Focus}

This preliminary phase invites test users to focus on the investigation domain, in particular by thinking of the elements. Elements are a selection of objects relevant to the topic/domain. In our analysis, we have chosen as elements 8 major game genres: Adventures, Racers, First-Person Shooters (FPS), Real-Time Strategy games (RTS), 
Sports, Arcades, Virtual Worlds (VW), and Role-Playing Game (RPG). The PCT highlights that a person makes sense of the world by experimenting and observing. It is very important that each participant focus on the genres using her personal experience. For this reason, participants are asked to select a well known VG for each element (genre) and to focus on it in the subsequent step.

\subsection{Construct Elicitation and Ratings}

We apply the triad method. Every participant ias interviewed by a researcher. Users are asked to identify one VG for each genre defined in the previous step. During the interview, the participant is invited to think about the $8 \mathrm{VG}$ genre samples in groups of three - this is the triading, in RGT's jargon. For every meaningful triad, the participant has to identify a property or a quality important enough to explain how two of the elements are similar, but different from the third one. The user has to put a label on that property. For instance, in the group of Quake (FPS), Warcraft (RTS), and Colin McRae Rally (Racers), one of the participants picked out Colin McRae, and described the property as "the Colin McRae situations, 3D world, settings and simulation are very realistic and credible, while Quake and Warcraft are fantasy", that we abstracted and added to the "likelihood" construct. This abstraction and cataloguing phase - namely, elicitation of the constructs - is described in detail in the 5.1 subsection.

For each one of the elicited constructs, the participant rates each one of the 8 elements (also those that not appear in the specific triad from which a particular construct was elicited) according to a five-graded scale, where 0 represents a low relevance of the property for the games in the triad, while 4 represents a high relevance.

\section{Evaluation of Videogame Player Experience}

This section presents the results obtained by the above presented RGT method. The data collected in the experiment consist of the personal constructs elicited by the participants and the rating of the investigated elements (VG genres) along such elicited constructs. The first data provide a qualitative insight about the characterization according to the user experience - of the investigated domain. The second data provide a quantitative measure of the degree of relevance of each elicited construct. This section describes the methodology for processing such data.

\subsection{Elicited Dimensions}

IRGT typically produces a large number of individual tables (we have collected a total of 570 constructs). Sample bipolar constructs include: "a good experience is necessary to play - it can be played without training", "the game requires cognitive effort it can be played also by a monkey". Differently than in clinical studies, we are not interested in the identification of idiosyncrasies. Instead, our research aims at finding out the basic and commonly shared criteria that players use in evaluating VGs. In our study each table contains a fixed number (8) of elements, a fixed number of constructs (we ask participants to consider ten triads) and a shared rating system (a bipolar fivegrade scale). From these shared features, it is possible to apply statistical methods 
(semantically blind methods) and/or semantic similarity (statistically blind methods) to analyze data interpersonally. In particular, we can search for patterns to compare constructs from all participants and assemble constructs showing some degree of similitude into groups.

We have analyzed the collected data and grouped the users' personal constructs into clusters. To this end, each pole of each construct was carefully semantically interpreted (using a discourse analysis technique) to capture the character of construct and to propose some shared labels - called dimensions - able to characterize all similar construct as a whole. The number of test users' personal constructs that have been grouped under a dimension is an indicator of the relevance of that dimension among the subjects (we call this the dimension weight $\mathrm{W}$ ). The higher is the number of grouped constructs, the

Table 1. Description of dimensions

\begin{tabular}{|c|c|c|}
\hline Dimensions & Descriptions & $\mathbf{W}$ \\
\hline Ability demand & $\begin{array}{l}\text { The game requires user's ability in terms of capabilities, skills and cogni- } \\
\text { tive efforts. }\end{array}$ & 94 \\
\hline \multirow[t]{2}{*}{$\begin{array}{l}\text { Dynamism } \\
\text { Style }\end{array}$} & $\begin{array}{l}\text { The activities in the game space are varied, unexpected and with frequent } \\
\text { and quick changes. }\end{array}$ & $\begin{array}{l}58 \\
48\end{array}$ \\
\hline & $\begin{array}{l}\text { The game has a distinctive appearance (modern, old) on the basis also of its } \\
\text { genre (a F1 race game should recreate a Grand Prix' atmosphere) }\end{array}$ & \\
\hline Engagement & $\begin{array}{l}\text { The game is exciting, challenging, charming, compelling. The player is } \\
\text { drawn never to stop the game. }\end{array}$ & 38 \\
\hline Emotional affect & $\begin{array}{l}\text { The game affects the player's emotional state. It induces anxiety, excite- } \\
\text { ment, and suspense. }\end{array}$ & 35 \\
\hline Likelihood & The game situations, 3D reconstructions are very realistic and credible. & 33 \\
\hline Sociality & The game mechanic allows the player to form social groups. & 28 \\
\hline Enjoyability & The game provides amusement, satisfaction, pleasure and gratification. & 28 \\
\hline Complexity & $\begin{array}{l}\text { The gameplay is composed by a huge amount of entangled experiences; the } \\
\text { virtual word to be explored is wide and with a great variety of landscapes. }\end{array}$ & 24 \\
\hline Playability span & $\begin{array}{l}\text { The game has the ability to involve players for long periods of time (e.g. } \\
\text { several months). }\end{array}$ & 23 \\
\hline $\begin{array}{l}\text { Technological } \\
\text { Quality }\end{array}$ & $\begin{array}{l}\text { The game is very accurate from the technological point of view: good } \\
\text { graphics, sound, multimedia contents, GUI design. }\end{array}$ & 23 \\
\hline Plot & The game is fascinating because the plot is very intriguing. & 21 \\
\hline Physical effort & $\begin{array}{l}\text { The game is very demanding in terms of physical activities (stress, muscu- } \\
\text { lar tension, movements of muscles). }\end{array}$ & 20 \\
\hline Replayability & $\begin{array}{l}\text { The game experience is varied enough (in terms of plot or mechanic) for the } \\
\text { player to repeat it several times without exhausting all possibilities. }\end{array}$ & 18 \\
\hline Controllability & $\begin{array}{l}\text { Players are given large freedom in selecting actions, interacting with the } \\
\text { ambient and configuring options. The player's avatar is highly customiza- } \\
\text { ble, also concerning its personality. }\end{array}$ & 15 \\
\hline Identification & $\begin{array}{l}\text { The player controls a character with a specific role in the game plot and } \\
\text { she/he feels a sense of identification with the character. }\end{array}$ & 13 \\
\hline Open-end & $\begin{array}{l}\text { The game plot is almost endless. The player is involved in long stories that } \\
\text { continue indefinitely. }\end{array}$ & 12 \\
\hline $\begin{array}{l}\text { Active involve- } \\
\text { ment }\end{array}$ & $\begin{array}{l}\text { The player is an active subject in the game and she/he plays also a creative } \\
\text { role. For example, she can build her own 3D models in the virtual world, or } \\
\text { modify the character's aspect with personal contents. }\end{array}$ & 10 \\
\hline $\begin{array}{l}\text { Competition/ } \\
\text { cooperation }\end{array}$ & $\begin{array}{l}\text { The game gives high relevance to the score. It offers possibilities to cooper- } \\
\text { ate or compete with other players towards a common goal. }\end{array}$ & 10 \\
\hline
\end{tabular}


more relevant is the cluster and higher is its weight, as more test users have highlight it. To reduce the risk of possible biases or misunderstandings in the definition of dimensions we have selected labels among existing personal constructs rather than inventing new ones. As an example of this labeling work, the dimension "Ability" takes into account 94 personal constructs. We have selected the term "ability" because it is the most frequent used in the participants descriptions of the related constructs. The 23 dimensions that resulted can be regarded as the most pertinent features of the participants' understandings of the VGs genres considered in the study and a list with the most important dimensions is provided in the table 1.

Analyzing the quantitative data on dimensions, we can gain significant insights into factors that players consider key in characterizing their gaming experience.

First, we see that VGs are perceived as engaging challenges where personal abilities are continuously put to the test, in particular with emotional involvement and frequent and quick changes in context and situation. This highlights the reactive nature of VGs and stresses the fact that players like being stimulated and testing/showing/training their reaction capabilities, also in a mechanical - almost non conscious - way, as it emerged from some informal comments we recorded during the (e.g. "I play to distract myself", "When I play I don't want to think"). It has to be noted that, despite users declare they like variety and change, they do not explicitly report the repetitive nature of several of the most popular games (e.g. shoot'em up and some sport games), which is apparent to the researchers. We may argue that players like intrinsic repetitive patterns (e.g. shooting, firing, jumping, running, etc.) but they want them to be applied to several different contexts and situations, and at different levels of difficulty. We may assimilate this to a basic, easy to learn alphabet that has to be used in a variety of contexts to tell a variety of tales.

Plot and sociality are not so important. This highlights that users are not particularly interested in these aspects and that the currently most successful VGs do not strongly support these dimensions.

Distinctive style and multimedia quality are relevant evaluation parameters, which highlights the importance of design and technological excellence.

The dimensions that we have elicited from the user tests tend to include the heuristics normally used by professionals and players when writing game reviews in specialized magazines and web sites (e.g. [23]), that are quite focused on the game interface and mechanics [24]. Other heuristics are pointed out in academic works, such as the well established GameFlow approach [25] [26], and underline the importance for a game to involve the player through immersion and concentration-keeping challenges. The dimension list from our tests tends to include the heuristics of both the above mentioned typologies and has two important features: it gives a weight to the relevance of the various dimensions and is based on the experience of real users rather than on psychological theories or on the practice and knowledge of a professional user or reviewer.

\subsection{Assessing Videogame Genres along the Elicited Dimensions}

The subsequent step of test consists in the users rating in a 0-4 scale of each VG genre along each one of the elicited dimensions. The data collected from each single test participant is then summarized in a table - namely, the Repertory Grid - that reports the average value of the personal tables. Table 2 shows the RG for our case. 
Table 2. Repertory Grid for the evaluation of VG genres along the elicited dimensions

\begin{tabular}{lcccccccc}
\hline & Adv & Racer & FPS & RTS & Sport & Arcade & VW & RPG \\
\hline Sociality & 2,50 & 2,89 & 2,93 & 3,38 & 4,00 & 2,26 & 2,83 & 2,56 \\
Open-end & 1,00 & 3,56 & 2,75 & 3,00 & 3,20 & 2,67 & 1,75 & 3,50 \\
Likelihood & 2,31 & 3,06 & 3,29 & 2,89 & 3,14 & 3,00 & 4,00 & 2,72 \\
Dynamism & 3,79 & 3,51 & 3,64 & 2,64 & 3,36 & 3,62 & 3,48 & 3,12 \\
Ability Demand & 3,14 & 2,88 & 3,43 & 3,54 & 3,20 & 2,80 & 2,74 & 3,51 \\
Emotional Affect & 3,06 & 3,04 & 3,55 & 2,71 & 2,46 & 2,81 & 2,33 & 3,79 \\
Active Involvement & 3,14 & 2,20 & 4,00 & 3,00 & 2,57 & 2,43 & 3,67 & 4,50 \\
Competition Cooperation & 1,00 & 2,14 & 3,44 & 3,13 & 3,75 & 2,38 & 3,00 & 3,00 \\
Identification & 3,44 & 3,00 & 2,50 & 1,80 & 3,08 & 3,43 & 4,75 & 3,33 \\
Plot & 3,31 & 2,62 & 3,27 & 3,67 & 2,67 & 1,82 & 3,00 & 3,43 \\
Re-playability & 2,33 & 3,13 & 3,38 & 3,71 & 3,33 & 2,88 & 3,67 & 4,08 \\
Playability Span & 3,60 & 3,24 & 3,33 & 3,45 & 3,86 & 2,93 & 4,00 & 3,45 \\
Controllability & 2,50 & 3,00 & 2,88 & 3,33 & 3,43 & 2,33 & 3,67 & 3,33 \\
Complexity & 2,45 & 3,20 & 2,94 & 3,17 & 3,25 & 2,55 & 3,60 & 4,15 \\
Style & 2,75 & 3,59 & 2,77 & 3,04 & 3,16 & 2,45 & 2,88 & 3,45 \\
Tecnological Quality & 2,62 & 3,44 & 3,82 & 3,29 & 3,62 & 2,33 & 4,55 & 3,30 \\
Physical Effort & 3,17 & 3,43 & 3,10 & 3,83 & 3,12 & 2,80 & 3,22 & 3,14 \\
Engagement & 3,67 & 3,48 & 3,53 & 3,81 & 3,08 & 3,16 & 3,43 & 3,71 \\
Enjoyability & 3,67 & 3,91 & 3,21 & 3,50 & 3,43 & 4,14 & 3,44 & 3,60 \\
\hline
\end{tabular}

\subsection{Results at a Glance}

While, the RG table provides the detail of the quantitative analysis, an alternative presentation method may be used, especially in order to provide a more immediate visualization of the results. This method exploits a radar graph. The graph is consists of a two-dimensional chart with several equi-angular axes - called radii - one for each dimension. An element (VG genre) is represented by its values shown on each radium of the graph. For each element, a line is drawn connecting the values, obtaining a starlike graph. If the number of elements is large, the graph tends to become cluttered, especially if printed in black and white. Figure 1 reports the radar graph for just 4 elements of our analysis (FPS, RPG Adventures and VW). However, the color graph representing all the 8 investigated elements is interesting in order to have a complete outlook of the domain, and is available online ${ }^{1}$.

As anticipated, the graphical representation is particularly useful to grasp an immediate idea of the analysis, suggesting immediate answers on issues that are relevant to game designers and evaluators, such as: what dimensions are prevalent for a given genre? What genres rely on a particular user construct? Which genres are similar/different among each other, and why? Are there some outliers? We will discuss these questions in the next subsection.

\subsection{Interpretation of the RG Results}

Observing the radar plot it is possible to get information about the single elements or identify clusters of game genres with similar features. For instance, looking at the star plot of the Virtual World genre we can see that is characterized by a star plot with

\footnotetext{
${ }^{1}$ www.elios.dibe.unige.it lradar.jpg
} 
peaks in single dimensions. It is the most technological demanding genre, is rated below the average concerning the physical effort and scores high in playability span, identification and likelihood. Other genres, instead, have a more balanced star graph. We expect that genres with a balanced graph tend to provide the player with a more "complete" experience. In effect, FPSs, that are the most common and popular games at present - in the sense that they are played by almost every videogamer, even if they are not necessarily the most appreciated type - have a graph without particular peaks. On the other hand, genres with a sparked star plot (e.g. VWs, RPGs) are likely to be particularly appealing to more specialized types of player.

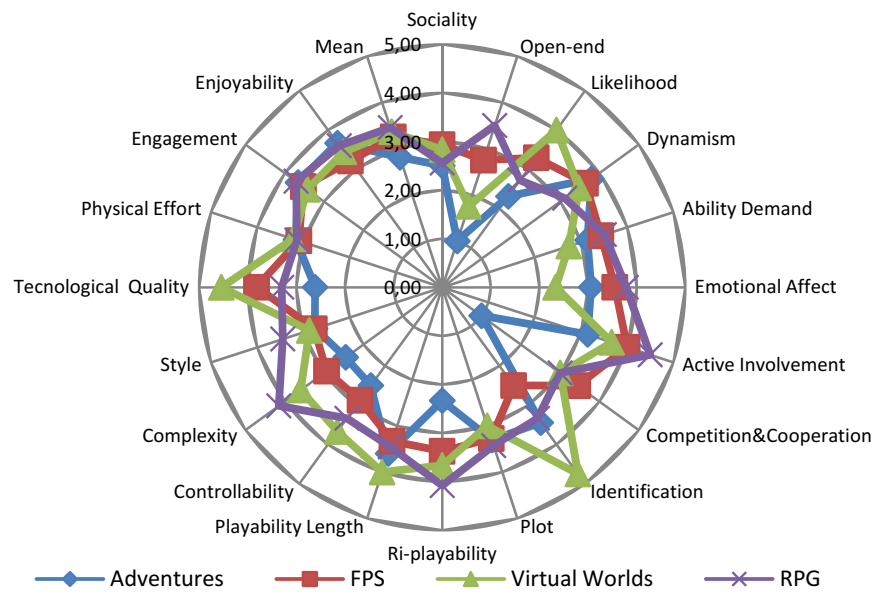

Fig. 1. The radar plot of games genres against genres

In an adventure VG, the player apparently likes plunging her/himself in the adventure defined by the author. In fact, the plot dimension has a significant value. Correspondingly, the adventure genre has the lowest score in the "open-end" dimension. We can argue that, for an adventure, an intricate and meaningful story is the most important feature and it is difficult for a game to combine such a type of plot with an open-end. Likelihood, on the other hand, is not a decisive factor for an adventure - in fact we have a number of successful adventures that involve fantasy settings. While likelihood is important for VWs, probably because the users like having clear, familiar references in a VW where a pre-defined, authored plot is not present and players have to build meaning and get enjoyment from their interaction among each other and with the environment. We argue that results may suggest that the lack of plot has to be compensated also by high technological quality (multimedia and GUI) as well.

Observing the single dimensions, we can observe, that the most important one according to the dimension analysis in tab. 1 - namely "ability demand", is characterized by a very little variance among the elements. This means that all types of VGs have to be strong in such dimension in order to appeal to the audience. This is true also for all the other important dimensions, such as dynamism, style, engagement. This spurs us to argue a generalization. That is that all the VGs, independent of their 
genre, have to provide some main ingredients to be successful. VG design has few niches, suggested by peaks in the graph.

Player perception of RPGs stresses the importance of complexity and active involvement. In fact, state of the art RPGs are characterized by a huge number of rules, cards and objects. The player has a lot of things to understand, learn and remember.

For VWs, the identification dimension is quite significant. This is reasonable since VW players have to differentiate among each other to be distinct in the VW. This is also already supported by some state of the art features, such as the customization of the avatar through the choice of glasses, hat, cloths, character aspects, etc. We have already seen some important distinctive factors for VWs (likelihood, technological quality, long span playability, identification). Generalizing, we can see that VWs (and RPGs, to a lesser extent) tend to require some more specialized features for game design.

Combining information from the dimension analysis (table 1) and the radar graph is interesting to think of new "hybrid" game genres. In particular, we try to combine game genres that have peaks in the most important dimensions - given for granted that, as we have seen, the most popular dimensions (e.g. "ability demand") are genreindependent and thus have to be targeted in any type of game design. Dynamism (the second highest weight in table 1) is important in particular for adventures, while style (the third weight) for racers and RPG. Combining adventures and RPGs could be of interest even if it is very challenging from an authorial point of view, since the game should combine a strong, compelling and meaningful plot (typical of adventures) with the possibility for every player of freely and actively interpreting her/his own role.

\section{Conclusions}

The scientific and industrial community related to the VG research and business is ever more concerned about the need for proper evaluation and assessment of games [4]. Devising suited methodologies is difficult, because the gaming experience is quite subjective and involves a number of different and possibly also conflicting aspects. This is apparent, for instance, as different game genres are now popular, that target different types of audience.

In this paper we have proposed an assessment methodology based on the PCT psychological theory and evaluated its validity in a test aimed at defining the features of a space that defines how state of the art commercial games are perceived by players. Results highlight that VGs are perceived as engaging challenges where personal abilities are continuously put to the test. This stresses the reactive nature of VGs and the fact that players like being stimulated and developing and testing their reaction capabilities. The space defined from the our tests' dimension tends to include the heuristics of the two major typologies of game review/evaluation methodologies: the approach typically used by professionals and players, who are quite focused on the game interface and mechanics, and the academic work that stresses the importance of an immersive experience and of concentration-keeping challenges. PCT results add two important features. First, it provides a quantitative evaluation, by giving a weight to the relevance of each dimension. Second, it is based on the experience of real users rather than on psychological theories or on the practice and knowledge of experts. 
We have then analyzed how current game typologies/genres are positioned with respect to such dimensions. This led to a number of interesting characterizations. Generalizing, we observed that some typologies cover a number of dimensions (e.g. FPS), while other typologies have peaks in single dimensions (e.g. Virtual Worlds along technological quality, identification and likelihood). We argue that the former games tend to provide the player with a "complete" experience, thus should be more interesting in a medium-long term and for a larger audience. The latter typologies, on the other hand, should be particularly appealing to specialized and keen players. Understanding game typology characterization should have a significant relevance for the design of new games. In particular, we expect that successful games should have a similar characterization as their typology. This means that they should address in particular those player experience dimensions that are perceived as relevant for that specific typology. However, this is a speculation that has to be investigated with further testing. Finally, we have also tried to see if new, "hybrid" game typologies could be devised based on the combination of important constructs.

\section{References}

1. Barnes, T., Encarnação, L.M., Shaw, C.D.: Serious Games. IEEE Computer Graphics and Applications 29(2), 18-19 (2009)

2. Pine II, B.J., Gilmore, J.H.: Welcome to the Experience Economy. Harvard Business Review, 97 (July-August 1998)

3. Sliney, A., Murphy, D.: JDoc: A Serious Game for Medical Learning. In: Proc. International Conference on Advances in Computer-Human Interaction, pp. 131-136 (2008)

4. Bernhaupt, R., Eckschlager, M., Tscheligi, M.: Methods for evaluating games: how to measure usability and user experience in games? In: Proc. of the international Conference on Advances in Computer Entertainment Technology, Salzburg, Austria, June 13-15 (2007)

5. Bannister, D., Fransella, F.: Inquiring man, 3rd edn. Routledge, London (1985)

6. Hudson, R.: Images of the Retailing Environment: An Example of the Use of the Repertory Grid Methodology. Environment and Behavior 6, 470-494 (1974)

7. Hassenzahl, M., Trautmann, A.: Analysis of web sites with the repertory grid technique. In: CHI 2001 Extended Abstracts on Human Factors in Computing Systems, Seattle, Washington, March 31-April 5 (2001)

8. Mandryk, R.L., Atkins, M.S., Inkpen, K.M.: A continuous and objective evaluation of emotional experience with interactive play environments. In: Proc. of the SIGCHI Conference on Human Factors in Computing Systems, Montréal, Québec, Canada, April 22-27 (2006)

9. Kelly, G.: The psychology of personal constructs, vol. 1 \& 2. Routledge, London (1955)

10. Tan, F.B., Hunter, G.M.: The Repertory Grid technique: A Method For The Study of Cognition in Information Systems. MIS Quarterly 1(26), 39-57

11. Federoff, M.: Heuristics and usability guidelines for the creation and evaluation of fun in video games. Unpublished thesis, Indiana Univ., Bloomington

12. Sweetser, P., Wyeth, P.: GameFlow: a model for evaluating player enjoyment in games. Computers in Entertainment 3(3) (July 2005)

13. Raney, A.: Expanding disposition theory: Reconsidering character liking, moral evaluations, and enjoyment. Communication Theory 14(4), 348-369 (2004) 
14. Nabi, R., Krcmar, M.: Conceptualising media enjoyment as attitude: implications for mass media effects research. Communication Theory 4(14), 288-310 (2004)

15. Green, M., Brock, T., Kaufman, G.: Understanding media enjoyment: The role of transportation into narrative worlds. Communication Theory 14(4), 311-327 (2004)

16. Chen, J.: Flow in games (and everything else). Commun. ACM 50(4), 31-34 (2007)

17. Ranganathan, C., Ganapathy, S.: Key dimensions of business-to-consumer websites. Information \& Management 39, 457-465

18. Clarke, D., Duimering, P.R.: How computer gamers experience the game situation: a behavioral study. Comput. Entertain. 4(3), 6 (2006)

19. Steed, A., McDonnell, J.: Experiences with Repertory Grid Analysis for Investigating Effectiveness of Virtual Environments. In: PRESENCE 2003, October 6-8. Aalborg Univ. (2003)

20. Boyd, S., Carini, D., Carini, C.: Constructing a Player-centered definition of fun for video games design. In: Proceedings of HCI 2004, Leeds Metropolitan University, UK (September 2004)

21. Hazlett, R.L.: Measuring emotional valence during interactive experiences: boys at video game play. In: Proceedings of the SIGCHI Conference on Human Factors in Computing Systems, Montréal, Québec, Canada, April 22-27 (2006)

22. Yannakaki, N., Lund, H.H.: Entertainment capture through heart rate activity in physical interactive playgrounds. User Modeling and User-Adapted Interaction 18(1) (2008)

23. Larsen, J.M.: Evaluating User Experience - how game reviewers do it, Workshop on Evaluating User Experiences In Games. In: CHI 2008 Conf., Florence, Italy, April 5-10 (2008)

24. Bellotti, F., Berta, R., De Gloria, A., Zappi, V.: Exploring Gaming Mechanisms to Enhance Knowledge Acquisition in Virtual Worlds. In: 3rd International Conference on Digital Interactive Media in Entertainment and Arts (DIMEA 2008), Athens, Greece (September 2008)

25. Cowley, B., Charles, D., Black, M., Hickey, R.: Toward an understanding of flow in video games. Comput. Entertain. 6(2), 1-27 (2008)

26. Clarke, D., Duimering, P.R.: How computer gamers experience the game situation: a behavioral study. Comput. Entertain. 4(3), 6 (2006) 